\section{Nephrectomy waiting time up to 3 months does not affect survival in patients with RCC}

Clinical data on the influence that waiting time for nephrectomy has on outcomes in patients with renal cell carcinoma (RCC) are lacking, and clinicians do not have relevant guidelines for the appropriate interval between diagnosis and surgical intervention for RCC. Stec et al. now report the effect of waiting times for nephrectomy on survival outcomes.

The researchers retrospectively reviewed data on 655 patients who underwent partial or radical nephrectomy for $\mathrm{RCC}$ at a single institution between 1988 and 2006. For analyses, the cohort was grouped by surgical waiting time, which was defined as time from first presentation to the urologist to the date of surgical resection. Mean follow-up duration was 31 months (range 0-214 months).

Mean waiting time was 1.2 months and $94 \%$ of surgeries were performed within 3 months. Surgical waiting time did not affect overall or disease-free survival. After adjustment for tumor stage, grade and histology, 5-year recurrence-free survival was also unaffected by waiting time. On multivariate analysis, significant predictors of worse 5-year recurrencefree survival were T stage, grade, lymph-node involvement and histology. Patients who waited less than 1 month for surgery were more likely to have high-stage and high-grade tumors than those who waited longer.

The findings highlight that urologists' judgment is the most important factor in deciding the appropriate time frame for surgery for RCC (i.e. on the basis of tumor size), as waiting periods in the time frames assessed here did not affect survival.

Original article Stec AA et al. (2008) Waiting time from initial urological consultation to nephrectomy for renal cell carcinoma-does it affect survival? J Urol 179: 2152-2157

\section{Marginal donor kidneys benefit high-risk transplant candidates}

Elderly renal transplant candidates and those with multiple comorbidities might not survive the wait for a healthy kidney from a deceased donor, and suitable living donors often cannot be found. Nicol et al. report the use of a novel donor source that might increase the number of kidneys available for transplantation. Their study assessed the outcome of renal transplantation using kidneys from which small masses had been removed.

A total of 43 patients ( 3 had significant comorbidities, 40 were aged $>60$ years) underwent renal transplantation using kidneys from 3 deceased donors and 38 patients who had undergone radical nephrectomy. All donor kidneys had contained small renal tumors $(<3 \mathrm{~cm})$ presumed to be renal cell carcinoma, which were removed before transplantation. Transplant recipients were followed up with standard medical review and investigations, together with ultrasonography of the allograft every 3 months and chest X-rays. Only one episode of possible tumor recurrence was reported, which occurred 9 years after transplantation; this patient (age 71 years) refused surgical treatment but remained stable under observation throughout the study. All grafts maintained function during the follow-up period (median 25 months). Four patients died from unrelated illness.

The authors suggest that, in selected high-risk patients considered likely to die before a donor kidney becomes available, transplantation of kidneys from which small renal tumors had been excised could paradoxically prolong survival and increase quality of life. Such kidneys would normally be discarded as they do not meet the normal criteria for donor organs.

Original article Nicol DL et al. (2008) Kidneys from patients with small renal tumours: a novel source of kidneys for transplantation. BJU Int 102: 188-193 